EPITHELIAL CELL BIOLOGY

\title{
Absence of Fer protein tyrosine kinase exacerbates endotoxin induced intestinal epithelial barrier dysfunction in vivo
}

W Qi, K V J Ebbert, A W B Craig, P A Greer, D-M McCafferty

Gut 2005;54:1091-1097. doi: 10.1136/gut.2004.061887

Background and aims: Fer kinase is activated by a number of growth factors and cytokines, and phosphorylates cortactin during cell shape change induced cortical actin reorganisation. In addition, Fer participates in cytoskeletal interactions mediated by cadherins, platelet endothelial cell adhesion molecule 1 (PECAM-1), and integrins, and has recently been implicated in limiting the innate immune response. Here we examined the role of Fer in modulating leucocyte recruitment and epithelial barrier function in the gut in response to lipopolysaccharide (LPS).

See end of article for Methods: Mice targeted with a kinase inactivating mutation (FerDR) or strain matched wild-type (129Sv/J) mice were studied after intraperitoneal injection of LPS. Intravital microscopy was used to examine intestinal leucocyte kinetics, and leucocyte infiltration was assessed by fluorescence activated cell sorting. Systemic inflammation was assessed by measuring lung myeloperoxidase activity. Epithelial barrier function was assessed in vivo using blood to lumen ${ }^{51} \mathrm{Cr}$-EDTA clearance, with or without antibody based depletion of circulating neutrophils.

Results: LPS induced a significant increase in leucocyte adhesion and neutrophil infiltration into the intestinal tissue, and increased blood to lumen ${ }^{51} \mathrm{Cr}$-EDTA clearance. Pretreatment with neutrophil depleting antibody completely abrogated this response in wild-type mice. In FerDR mice, LPS induced leucocyte adhesion within the intestinal venules was exacerbated and associated with a trend towards increased neutrophil transmigration relative to wild-type mice. Surprisingly, LPS induced epithelial barrier permeability was increased 2.5-fold in FerDR mice relative to wild-type mice, and this barrier defect was only partly attenuated by depleting circulating neutrophils by $>93 \%$.

Conclusions: Fer plays a role in regulating LPS induced epithelial barrier dysfunction in vivo through both neutrophil dependent and neutrophil independent mechanisms.

Revised version recei

19 January 2005

Accepted for publication

24 January 2005

ipopolysaccharide (LPS) or endotoxin (a component of the outer membrane of Gram negative bacteria) is a

-dominant bacterial antigen which is capable of activating a variety of cell types to release inflammatory mediators, induce leucocyte infiltration, and cause mucosal dysfunction. Infections involving LPS continue to be a major clinical problem resulting in complications such as multiple organ dysfunction, septic shock, and mortality. ${ }^{1}$ Neutrophil recruitment from the microcirculation is a hallmark feature of the innate immune response and is the firstline of defence in the host's normal response to infection. However, in the gut, neutrophil recruitment has been directly associated with tissue damage and epithelial barrier dysfunction in response to various stimuli. ${ }^{2-6}$ Therefore, regulation of leucocyte recruitment and epithelial barrier integrity in response to toxins such as LPS, is essential in maintaining homeostasis in the gut.

Fer (Fes related) is a ubiquitously expressed cytoplasmic protein tyrosine kinase (PTK) belonging to the group IV subfamily of PTKs, ${ }^{78}$ which also includes the closely related Fps/Fes PTK. ${ }^{9}$ Fer kinase is activated by a number of extracellular stimuli, including epidermal and platelet derived growth factor, ${ }^{10}{ }^{11}$ insulin, ${ }^{12}$ erythropoietin, ${ }^{13}$ stem cell factor, ${ }^{13}{ }^{14}$ collagen and thrombin ${ }^{15}$ and on aggregation of the high affinity IgE receptor Fc $\in$ RI. ${ }^{14}{ }^{16}$ Fer is also activated by cell shape changes, ${ }^{17}$ leading to phosphorylation of cortactin, an important regulator of the cortical actin cytoskeleton. ${ }^{11}{ }^{17}{ }^{18}$ Fer has been implicated in the regulation of adherens junctions and crosstalk to focal adhesions. ${ }^{19-21}$ At adherens junctions, Fer and the tyrosine phosphatase, phosphotyrosine phosphatase $1 \mathrm{~B}$, cooperatively regulate the tyrosine phosphorylation status of cadherin bound $\beta$-catenin, ${ }^{22}$ which plays a key role in connecting adherens junctions to the cytoskeleton. At focal adhesions, Fer regulates the phosphotyrosine status of the scaffolding proteins pl30Cas and pl20 catenin. ${ }^{19} 2022$ Overexpression of Fer in fibroblasts diminished integrin based cell adhesion ${ }^{19}$ while Fer deficient mast cells displayed increased adhesiveness and reduced motility in response to FceRI engagement. ${ }^{14}$ These Fer deficient mast cell phenotypes correlated with diminished p38 activation. ${ }^{14}$ Fer has also been localised with newly assembling microtubules in protrusions at the leading edge of motile endothelial cells and was implicated in the phosphorylation of the cell-cell adhesion molecule platelet endothelial cell adhesion molecule 1 (PECAM-1). ${ }^{23}$ Fer mediated phosphorylation of PECAM-1 may play an important role in regulating signalling in vascular endothelial cells ${ }^{23}$ or diapedesis of activated leucocytes. ${ }^{24}$ PECAM-1 deficient mice display an enhanced innate immune response to LPS challenge. ${ }^{25}$ Interestingly, we have recently reported that Fer deficient mice display enhanced recruitment of leucocytes to skeletal muscle in response to LPS challenge. ${ }^{26}$

Abbreviations: LPS, lipopolysaccharide; PTK, protein tyrosine kinase; MPO, myeloperoxidase; Fer, fes related; FerDR, mice homozygous for the kinase inactivating D743R mutation; FACS, fluorescence activated cell sorting; ${ }^{51} \mathrm{Cr}$-EDTA, 51-chromium ethylenediamine tetra-acetic acid; PECAM-1, platelet endothelial cell adhesion molecule 1; $L_{50}$, lethal dose that kills $50 \%$ of group; $\mathrm{PE}$, phycoerythrin 
Taken together, these studies suggest key roles for Fer in growth factor and cytokine signalling, cell-cell and cellmatrix interactions, cell migration, and cytoskeletal remodelling. All of these cellular functions are important in the innate immune response. To date, the role of Fer kinase in the innate immune response in the gastrointestinal tract is not known. In this study, we evaluated the in vivo role of Fer in gastrointestinal inflammation induced by LPS. Fer deficient mice displayed an enhanced inflammatory response to LPS which correlated with increased leucocyte adhesion to endothelial cells in the gut microvasculature, and a striking neutrophil dependent epithelial barrier dysfunction. These observations provide compelling new evidence for a physiological role for Fer in regulating innate immunity in the gastrointestinal system.

\section{MATERIALS AND METHODS \\ Animals}

Fer kinase was inactivated in mice by targeting the fer locus with a kinase inactivating missense mutation ( $\mathrm{Fer}^{\mathrm{D} 743 \mathrm{R})}$ ), as previously described. ${ }^{11}$ In addition to abolishing kinase activity, the D743R mutation was found to destabilise the Fer protein, leading to its rapid degradation. Mice homozygous for the FerD743 mutation therefore behave essentially as Fer null animals. FerDR mice (maintained in a $129 \mathrm{~Sv} / \mathrm{J}$ background) were rederived by Jackson Laboratories and a colony established at the University of Calgary. Homozygous mutant mice (FerDR) are viable, fertile, and have no obvious phenotypic differences from wild-type mice. FerDR and wildtype mice were bred in the same facility and used between 6 and 10 weeks of age. All experimental procedures were approved by the University of Calgary Animal Care Committee and conform to guidelines established by the Canadian Council for Animal Care.

\section{Systemic LPS administration}

LPS $(0.5 \mathrm{mg} / \mathrm{kg})$ from Escherichia coli 0111:B4 (Biosciences Inc., La Jolla, California, USA), containing less than $0.8 \%$ contaminating bacterial proteins, was administered intraperitoneally. This dose of LPS has been previously used to examine leucocyte-endothelial interactions in vivo ${ }^{27}$ and is approximately $\mathrm{l} / 50$ of the $\mathrm{LD}_{50}$ (lethal dose that kills $50 \%$ of the group) for mice. ${ }^{28}$ Small intestinal intravascular leucocyte kinetics, epithelial barrier integrity, and leucocyte migration in wild-type or FerDR mice were performed 3.5-4.5 hours post intraperitoneal injection of LPS or vehicle as described below. In separate experiments, mice were treated with LPS doses of $0.005,0.05,0.5$, or $5 \mathrm{mg} / \mathrm{kg}$ and leucocyte kinetics were observed.

\section{Neutrophil depletion}

Mice were depleted of circulating neutrophils by a single intraperitoneal injection of $150 \mu \mathrm{g}$ of monoclonal antineutrophil antibody RB6 8C5 (Anti-Ly6G; BD Biosciences Pharmingen, Mississauga, Ontario, Canada) 24 hours prior to analysis. Manual counts (performed as described below) showed that circulating neutrophils were depleted by $>93 \%$ within 24 hours in both wild-type and FerDR mice. For depletion of tissue neutrophils, three injections of $150 \mu \mathrm{g}$ of RB6 8C5 were given at 24 hour intervals, and mice were analysed 24 hours after the final dose. This treatment regimen is sufficient to deplete resident and circulating neutrophil populations. ${ }^{42}$

\section{Circulating leucocyte counts}

Whole blood was obtained via cardiac puncture and total leucocyte counts were performed using a Bright-line haemocytometer (Hausser Scientific, Horsham, PA). Leucocyte differential counts were determined by examination of blood smears stained using harleco hemacolour kit (EM Science Gibbstown, New Jersey, USA).

\section{Intravital microscopy}

Small intestinal microcirculation was examined by intravital microscopy using a previously described method ${ }^{30}$ with slight modifications. Briefly, mice were anaesthetised and maintained on inhalation anaesthetic (isoflurane; Bimeda-MTC, Cambridge, Ontario, Canada). A jugular cannulation was performed for administration of rhodamine $6 \mathrm{G}$ and body temperature was maintained at $37^{\circ} \mathrm{C}$. A segment of small intestine was draped over a viewing pedestal and the submucosal vessels of the terminal ileum were observed using a microscope (Mikron Instruments, Inc., California, USA) with a $20 \times$ objective lens (Zeiss Achroplan $20 \times / 0.5 \mathrm{~W}$ ) and a periplan $10 \times$ eyepiece (final magnification $1500 \times$ ). Leucocytes were stained in vivo by intravenous injection of 0.05\% rhodamine 6G (Sigma-Aldrich Co., Missouri, USA). Images of the microcirculatory bed were recorded using a video camera (Pieper GMBH, Berlin, Germany) and a video recorder (Panasonic AG-1980 P; Matsushita Electric Ind. Co, Ltd, Osaka, Japan) over a 30 minute time frame. Five post capillary venules (diameter $25-45 \mu \mathrm{m}$ ) were selected in each mouse for study. Rolling leucocytes were defined as those white blood cells moving at a velocity less than that of blood flow in the same vessel. Leucocyte rolling velocity was determined as the time required for a leucocyte to traverse $100 \mu \mathrm{m}$ of venule, and averaged for 20 leucocytes $(\mu \mathrm{m} / \mathrm{s})$. Leucocyte flux was calculated as the number of rolling cells crossing a given point per minute (cells/minute). Leucocyte adhesion was quantified as the number of leucocytes that
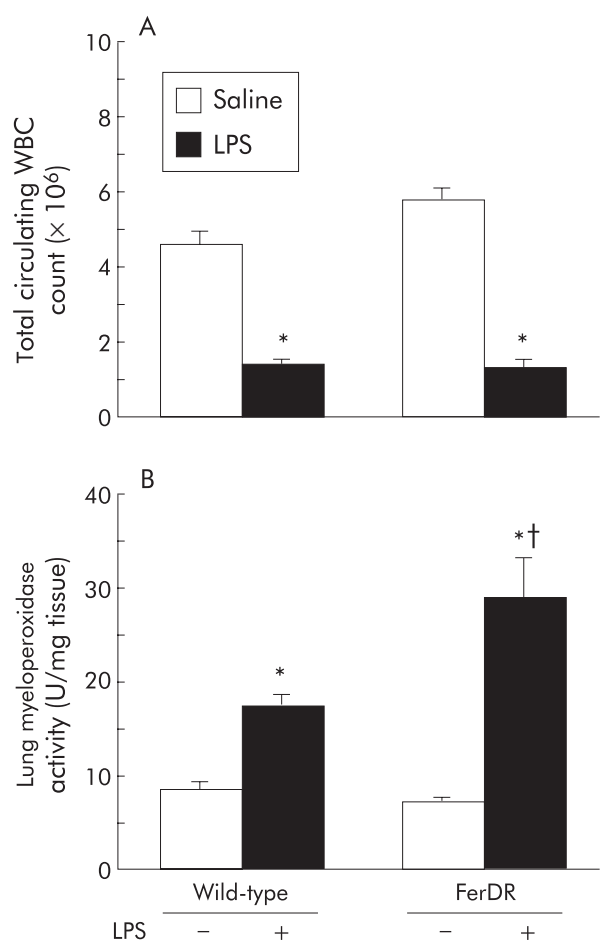

Figure 1 Systemic effect of intraperitoneal lipopolysaccharide (LPS) on circulating leucocyte counts and leucocyte recruitment to the lung.

(A) Total circulating blood leucocyte count and (B) lung myeloperoxidase activity four hours post intraperitoneal challenge with $0.5 \mathrm{mg} / \mathrm{kg}$ LPS or $0.9 \%$ saline in wild-type and FerDR mice. Values are expressed as mean (SEM); $n=3-6$; ${ }^{*} p<0.05$, significantly different from saline treated wildtype or FerDR mice; †significant increase over LPS challenged wild-type mice. 
remained stationary on the vessel wall for 30 seconds or more within a $100 \mu \mathrm{m}$ section (cells $/ 100 \mu \mathrm{m} / \mathrm{min}$ ).

\section{In vivo epithelial barrier function}

Epithelial barrier function was quantified by measuring blood to lumen 51-chromium ethylenediamine tetra-acetic acid $\left({ }^{51} \mathrm{Cr}\right.$-EDTA $)$ movement in the mouse small intestine, as previously described. ${ }^{31}$ Briefly, mice were anaesthetised by intraperitoneal injection with a cocktail of $10 \mathrm{mg} / \mathrm{kg}$ xylazine (MTC Pharmaceuticals, Cambridge, Ontario, Canada) and $200 \mathrm{mg} / \mathrm{kg}$ ketamine hydrochloride (Rogar/STB Inc., Montreal, Quebec, Canada). A jugular cannulation was performed to administer ${ }^{51} \mathrm{Cr}$-EDTA and the anaesthetic, as needed, and both renal pedicles were ligated to prevent excretion of ${ }^{51} \mathrm{Cr}$-EDTA into urine. A loop of small intestine was fitted with inflow and outflow tubes, and continuously perfused with warmed Tyrodes solution $(0.25 \mathrm{ml} / \mathrm{min})$. Samples of luminal perfusate were collected over 10 minute intervals for 60 minutes and ${ }^{51} \mathrm{Cr}$-EDTA activity was determined in plasma and luminal perfusate using an LKB CompuGamma spectrometer. Mucosal permeability was calculated as the plasma to lumen clearance of ${ }^{51} \mathrm{Cr}$-EDTA using the following calculation:

Clearance $=\mathrm{cpm}_{\mathrm{p}} \times \mathrm{pr} \times 100 / \mathrm{cpm}_{\mathrm{pl}} \times \mathrm{wt} \mathrm{ml} / \mathrm{min} / 100 \mathrm{~g}$ where $\mathrm{cpm}_{\mathrm{p}}$ is counts/min/ml in perfusate, pr is perfusion rate in $\mathrm{ml} / \mathrm{min}, \mathrm{cpm}_{\mathrm{pl}}$ is counts $/ \mathrm{min} / \mathrm{ml}$ in plasma, and wt is weight of the intestinal segment in grams.
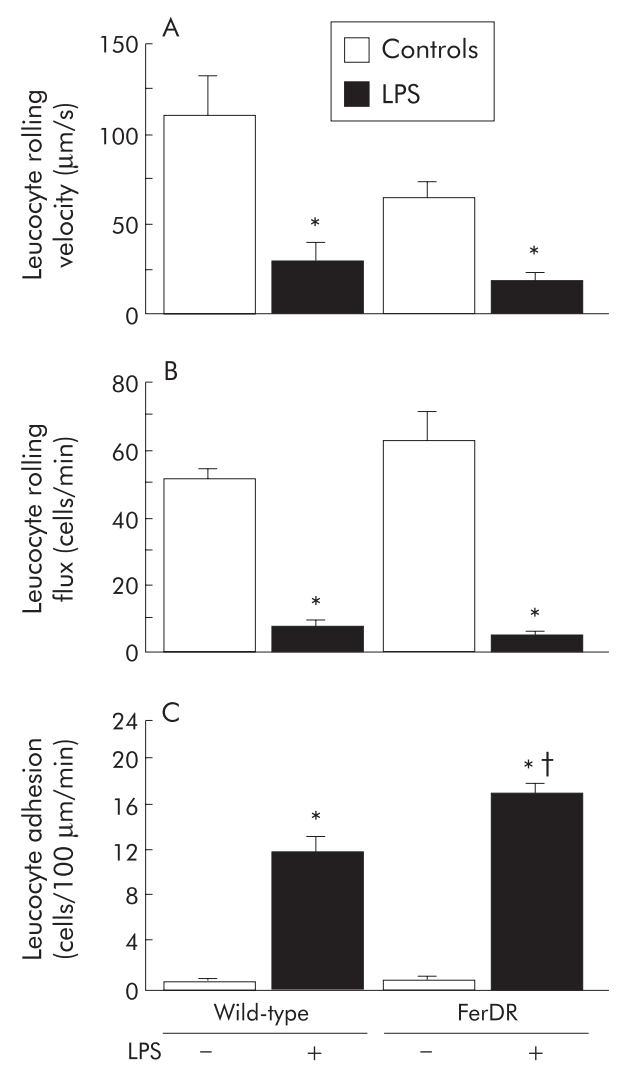

Figure 2 Lipopolysaccharide (LPS) induced leucocyte adhesion is enhanced in Fer deficient mice. Leucocyte kinetics in small intestinal submucosal venules: (A) leucocyte rolling velocity, (B) leucocyte rolling flux, and (C) leucocyte adhesion in wild-type or FerDR untreated or LPS treated mice. Data were obtained 3.5-4.5 hours post challenge with $0.5 \mathrm{mg} / \mathrm{kg}$ LPS intraperitoneally. Values are expressed as mean (SEM); $\mathrm{n}=6$ (exception, FerDR untreated group $\mathrm{n}=2$ ). * $\mathrm{p}<0.05$, significantly different from untreated wild-type mice; $†$ significant increase over LPS challenged wild-type mice.

\section{Lung myeloperoxidase activity}

Samples of lung tissue were weighed, frozen on dry ice, and stored at $-20^{\circ} \mathrm{C}$ for no more than one week before analysis. Myeloperoxidase (MPO) activity was determined as previously described. ${ }^{31} 32$ One unit of MPO activity was defined as that degrading 1 micromole of hydrogen peroxide per minute at $25^{\circ} \mathrm{C}$ and expressed as units of MPO activity per milligram of tissue (U/mg tissue).

\section{FACS analysis of intestinal leucocytes}

Leucocyte populations were quantified by fluorescence activated cell sorting (FACS) analysis by a modified version of a previously described method. ${ }^{33}$ Briefly, mice were anaesthetised by intraperitoneal injection of a xylazine/ ketamine cocktail, and the vasculature flushed with cold phosphate buffered saline via transcardiac perfusion. The entire small intestine was excised and digested with collagenase type III (Sigma-Aldrich, Missouri, USA), and leucocytes from the lamina propria were isolated on Percoll gradients. Cells were stained with $0.5 \mu \mathrm{g}$ of fluorescein isothiocyanate labelled anti-Ly-6G (BD Biosciences Pharmingen), $0.2 \mu \mathrm{g}$ of phycoerythrin (PE) labelled antiCD3 (BD Biosciences Pharmingen), or $0.2 \mu \mathrm{g}$ PE-anti-F4/80 monoclonal antibodies (Cedarlane Laboratories Ltd, Hornby, Ontario, Canada) for neutrophil, lymphocyte, and monocyte populations, respectively. Two colour stained cell populations were analysed by a FACScan fluorescence analyser (BD, Mountain View, California, USA). Cell viability was assessed using Trypan blue exclusion, and was determined as $>90 \%$ for all isolated cell populations.

\section{Statistical analysis}

Data were expressed as the mean (SEM). Groups of data were compared using a non-parametric Mann-Whitney U test or Kruskal-Wallis ANOVA, followed by a Dunn's multiple comparisons post test.
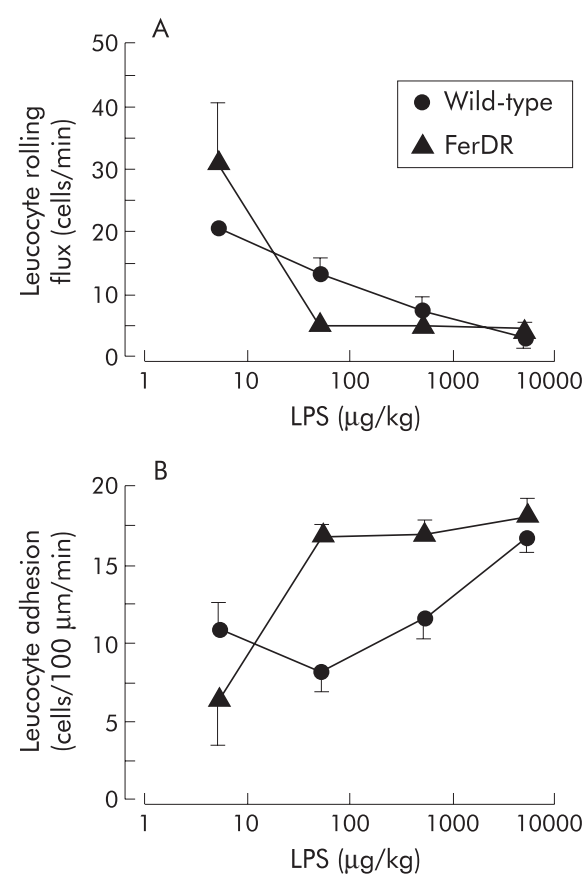

Figure 3 Maximal leucocyłe recruitment is achieved at lower concentrations of lipopolysaccharide (LPS) in the absence of Fer kinase. (A) Leucocyte rolling flux and (B) leucocyte adhesion in small intestinal venules of wild-type mice or FerDR mice challenged with $0.005,0.05$, 0.5 , or $5 \mathrm{mg} / \mathrm{kg}$ LPS. Vessels were studied $3.5-4.5$ hours post challenge. Data are expressed as mean (SEM); $n=2-6$. 


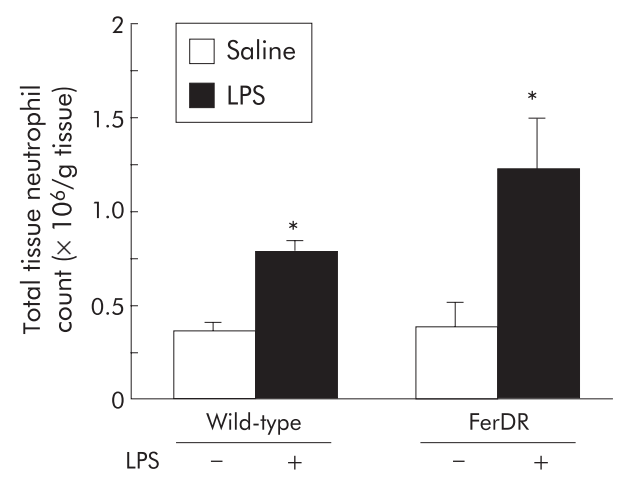

Figure 4 Neutrophil migration into intestinal tissue in response to systemic lipopolysaccharide (LPS). Total tissue neutrophil numbers were assessed by fluorescence activated cell sorting analysis, four hours post intraperitoneal challenge with $0.5 \mathrm{mg} / \mathrm{kg}$ LPS or saline in wild-type and FerDR mice. Values are expressed as mean (SEM); $n=3-4 ;{ }^{*} p<0.05$, significant increase from saline treated wild-type or FerDR mice.

\section{RESULTS}

Systemic response to intraperitoneal LPS challenge in wild-type and FerDR mice

To address the potential role of Fer in regulating the inflammatory response, we first assessed the systemic response observed after intraperitoneal LPS challenge. Total circulating white blood cell and differential analysis were similar in healthy wild-type and FerDR mice, as previously reported. ${ }^{26}$ Four hours after intraperitoneal LPS, a systemic response was observed in wild-type mice, as illustrated in fig 1. LPS challenge $(0.5 \mathrm{mg} / \mathrm{kg})$ induced a significant decrease in total circulating leucocyte counts in both wildtype and FerDR mice (fig lA). The drop in circulating leucocyte counts could be accounted for in part by increased accumulation of neutrophils in the lung, as indicated by increased lung MPO activity (fig $1 \mathrm{~B}$ ). Interestingly, a significantly greater LPS induced increase in lung MPO activity was observed in FerDR compared with wild-type mice (fig 1B), suggesting more robust recruitment of granulocytes.

\section{LPS induced leucocyte adhesion is enhanced in the absence of Fer kinase}

We next examined the behaviour of leucocytes within the submucosal venules of the small intestine using intravital fluorescence microscopy. In wild-type mice, LPS challenge induced a significant reduction in the number and velocity of rolling cells due to leucocyte sequestration in the lung and upregulation of adhesion molecules on endothelial cells, ${ }^{34}$ respectively; a concomitant significant increase in leucocyte adhesion was observed (fig 2). In FerDR mice, similar reductions in leucocyte rolling flux and velocity, and increased adhesion were observed after LPS challenge. Interestingly, the LPS induced increase in leucocyte adhesion was significantly greater in FerDR mice $(30 \%$; $<<0.05)$ compared with wild-type animals. LPS induced leucocyte recruitment was shown to be dose dependent in both wildtype and FerDR mice; however, FerDR mice were two orders of magnitude more sensitive, requiring only $0.05 \mathrm{mg} / \mathrm{kg}$ to achieve a maximal response, compared with $5 \mathrm{mg} / \mathrm{kg}$ in wildtype mice (fig 3). At $5 \mathrm{mg} / \mathrm{kg}$, no significant difference in leucocyte rolling velocity or adhesion was observed between wild-type and FerDR mice. These data show that loss of Fer kinase activity correlates with increased association and adherence of leucocytes to the endothelial surfaces of intestinal venules following LPS challenge.
Table 1 Small intestinal tissue lymphocyte and monocyte numbers $\left(\times 10^{6} / \mathrm{g}\right.$ tissue $)$

\begin{tabular}{lll}
\hline Group & Lymphocytes & Monocytes \\
\hline Wild-type: saline challenge & $1.0(0.02)$ & $3.1(0.4)$ \\
Wild-type: LPS challenge & $1.7(0.1)^{*}$ & $2.7(0.1)$ \\
FerDR: saline challenge & $1.8(0.4)$ & $3.2(0.9)$ \\
FerDR: LPS challenge & $2.9(0.8)$ & $3.5(1.0)$ \\
\hline \multicolumn{4}{l}{$\begin{array}{l}\text { Lymphocytes and monocytes were identified by fluorescence activated } \\
\text { cell sorting analysis of digested small intestine, as described in the }\end{array}$} \\
methods, using anti-CD3 and anti-F4/80 monoclonal antibodies, \\
respectively. $\mathrm{n}=3-4$. \\
LPS, lipopolysaccharide; FerDR, mice homozygous for the kinase \\
inactivating D743R mutation. \\
Significant difference (* $\mathrm{p}<0.05)$ from saline challenged wild-type mice.
\end{tabular}

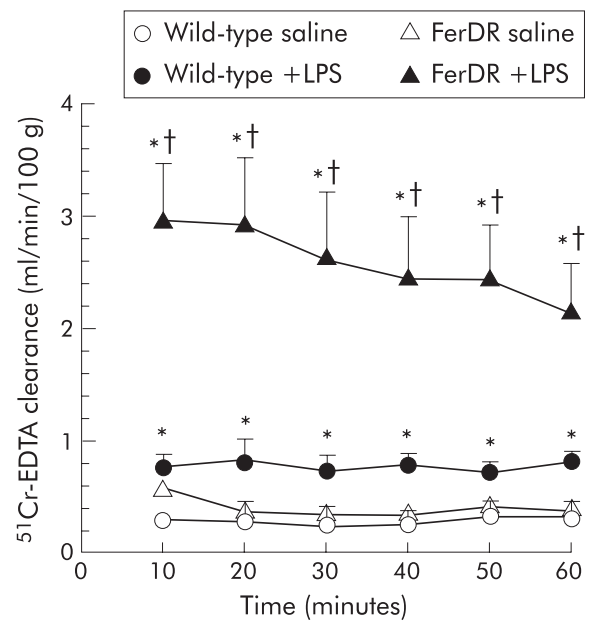

Figure 5 Lipopolysaccharide (LPS) induced mucosal barrier dysfunction in vivo is exacerbated in Fer deficient mice. Blood to lumen clearance of 51-chromium ethylenediamine tetra-acetic acid ( ${ }^{51} \mathrm{Cr}$-EDTA) was assessed in small intestinal loops as an indication of mucosal barrier integrity. Data were obtained for 10 minute intervals over a one hour period starting 3.5 hours post challenge with saline or $0.5 \mathrm{mg} / \mathrm{kg}$ LPS in wild-type or FerDR mice. Data are expressed as mean (SEM); $n=5-8$. ${ }^{*} p<0.05$ significant increase from saline treated wild-type or FerDR mice; †significant increase over LPS challenged wild-type mice.

LPS induced leucocyte migration in the small intestine We next examined the number of extravasated neutrophils in the small intestine in response to LPS challenge (fig 4). Wildtype mice had a significant increase in Ly-6 positive cells four hours post LPS challenge, suggesting a significant increase in neutrophil extravasation. Similar results were observed in FerDR mice, with a trend towards increased LPS induced neutrophil recruitment relative to wild-type mice. Small intestine MPO activities were lower than those observed in the lung (fig 1B), and significant increases after LPS treatment could not be determined (data not shown). This suggested that LPS induced neutrophil migration to the intestine was lower than to the lung. Lymphocyte numbers (CD3+ cells) were also significantly increased in wild-type mice after LPS challenge (table 1).

Although a similar trend was observed in FerDR mice, the LPS induced increase did not reach statistical significance. No significant increase in monocyte/macrophage numbers (F4/ 80 positive cells) were observed after LPS challenge in either wild-type or FerDR mice (table 1), suggesting monocytes were not recruited to the intestine. 

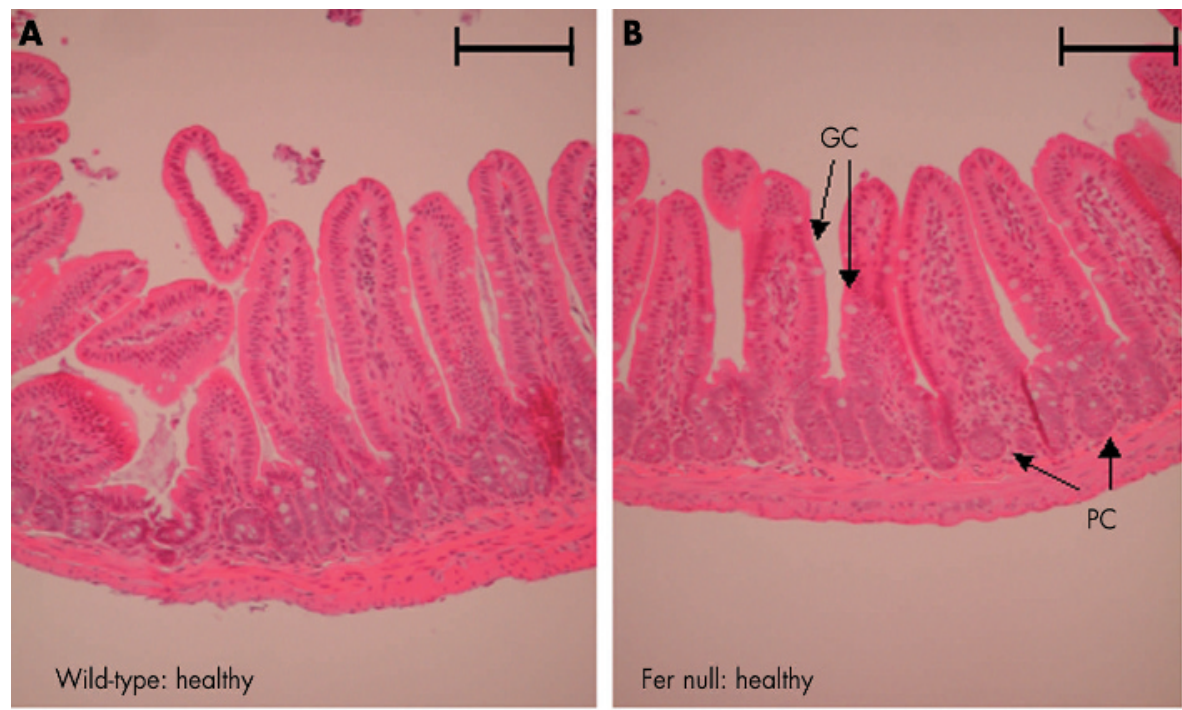

Figure 6 Lipopolysaccharide (LPS) induced mucosal damage is more severe in Fer deficient mice. Histological tissue sections from mouse small intestine stained with haematoxylineosin. Sections are representative of tissue taken from wild-type $(A, C ; n=5$ and 9) or FerDR (B, D; $n=4$ and 7) mice under normal conditions (A, B) or four hours post LPS treatment (C, D). GC, goblet cells; PC, Paneth cells; DE, denuded of epithelium. Magnification $140 \times$. Bar indicates $100 \mu \mathrm{m}$
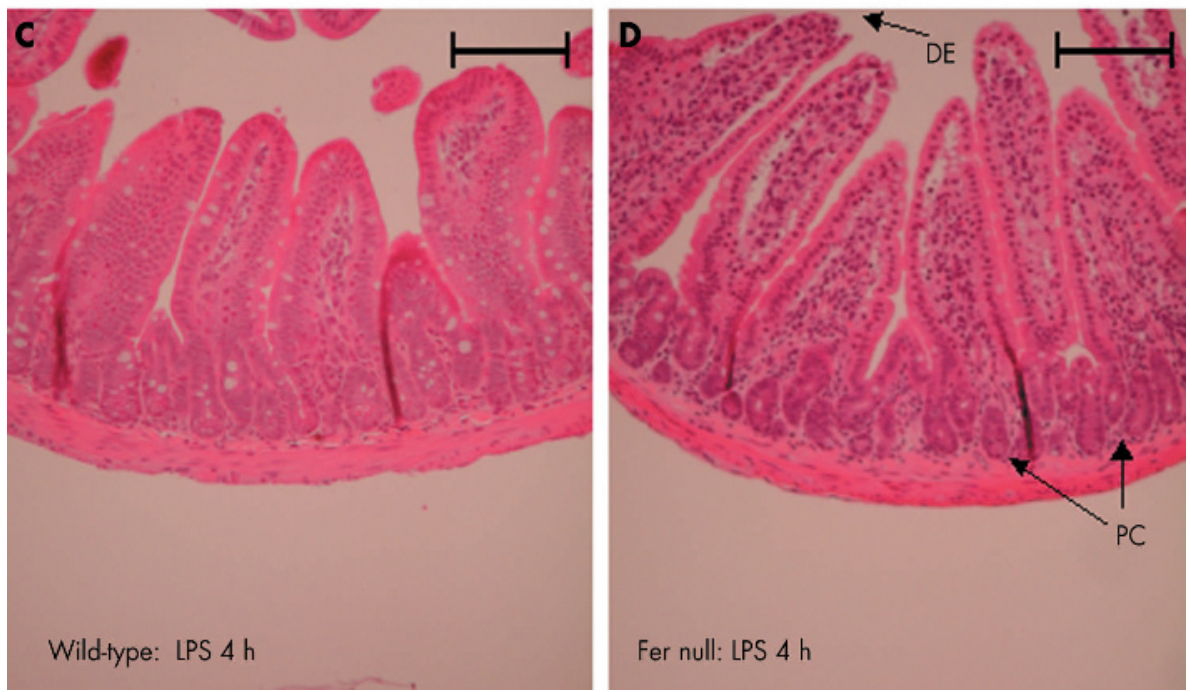

\section{LPS induced small intestinal epithelial barrier dysfunction is exacerbated in Fer deficient mice}

We next examined LPS induced changes in epithelial barrier integrity by measuring blood to lumen ${ }^{51} \mathrm{Cr}$-EDTA clearance for a one hour period, starting 3.5 hours after injection of LPS or saline (fig 5). In saline injected mice, blood to lumen clearance was maintained at an average of $0.3 \mathrm{ml} / \mathrm{min} / 100 \mathrm{~g}$ in both wild-type and FerDR mice. LPS challenge induced a significant (2.5-fold) increase in epithelial barrier dysfunction in wild-type mice 3.5 hours post challenge which was maintained for the duration of the experiment (one hour). Remarkably, a 7-10-fold increase in barrier dysfunction was seen in LPS challenged FerDR mice over the same period of time, which was significantly greater than LPS induced epithelial dysfunction in wild-type mice.

Histological analysis of the small intestine was undertaken and scores assigned according to the degree of cellular infiltration (0, 1, or 2; none, mild, or moderate) and the presence or absence ( 0 or 1 ) of goblet cell depletion, areas of sloughed epithelium, or Paneth cell depletion. In wild-type mice, LPS $(0.5 \mathrm{mg} / \mathrm{kg})$ administration correlated with a mild inflammatory response (fig 6C; compare with fig 6A, saline treated wild-type). In general, a normal mucosal architecture was observed with intact epithelial cell layer, mucus containing goblet cells, and evidence of a mild inflammatory infiltrate (mean histological score of $0.6(0.2)$ from $n=9$ ).
Both wild-type and FerDR mice without LPS had zero scores ( $\mathrm{n}=4$ and 5, respectively). In contrast, histological examination of LPS treated FerDR small intestine revealed a more significant inflammatory infiltrate and more fragile architecture (fig 6D; compare with fig 6B, saline treated FerDR). Generally, goblet cells and Paneth cells were depleted of their contents and there were areas denuded of epithelium, giving a mean histological score of $3.0(0.5)(n=7)$.

\section{Role of infiltrating neutrophils in LPS induced epithelial barrier dysfunction}

We next asked if neutrophils are required for LPS induced epithelial dysfunction in the mouse small intestine. This was achieved by depleting circulating neutrophils by pretreating mice with $150 \mu \mathrm{g}$ of antineutrophil antibody RB68C5 24 hours prior to LPS or vehicle challenge. In our hands, this treatment regimen depleted circulating neutrophils by $>93 \%$ in both wild-type and FerDR mice. In wild-type mice, neutrophil depletion completely abrogated LPS induced epithelial dysfunction (fig 7). ${ }^{51}$ Cr-EDTA clearance levels in LPS challenged neutrophil depleted wild-type mice were not significantly different from saline challenged controls, indicated by the broken line in fig 7 . These data demonstrate that in wild-type mice, LPS induced epithelial barrier dysfunction is mediated completely through infiltrating neutrophils. In FerDR mice, similar depletion of circulating 


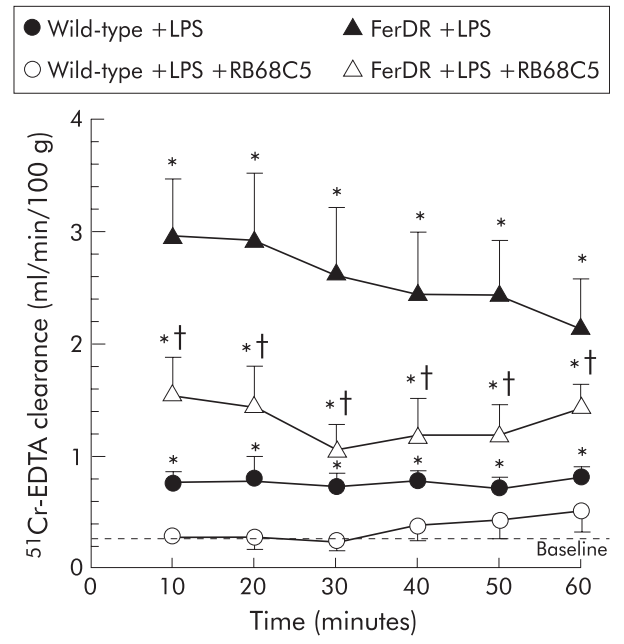

Figure 7 Neutrophil depletion completely abrogates lipopolysaccharide (LPS) induced epithelial barrier dysfunction in wild-type mice but only partially in Fer deficient mice. Epithelial barrier permeability represented by 51 -chromium ethylenediamine tetra-acetic acid $\left({ }^{51} \mathrm{Cr}-\right.$ EDTA) blood to lumen clearance, in wild-type mice and FerDR mice. Data were obtained at 10 minute intervals between 3.5-4.5 hours post challenge with LPS $0.5 \mathrm{mg} / \mathrm{kg}$ intraperitoneally. In separate groups, mice were treated with RB68C5 to deplete circulating neutrophils. Broken line indicates baseline permeability (saline treated, FerDR, or wild-type mice). Data are expressed as mean (SEM); $n=3$. * $p<0.05$, significant increase from saline treated FerDR or wild-type mice; $t p<0.05$ significant increase from RB68C5 treated wild-type mice.

neutrophils only inhibited LPS induced epithelial dysfunction by approximately $50 \%$; the remaining ${ }^{51} \mathrm{Cr}$-EDTA clearance level was significantly increased over saline treated control mice, suggesting that infiltrating neutrophils were only partly responsible for the LPS induced dysfunction in FerDR mice. This residual LPS induced mucosal dysfunction in FerDR mice was not reduced further by chronic treatment of mice with RB68C5, which depletes both circulating and tissue resident neutrophils (data not shown).

\section{DISCUSSION}

In this study we have described, for the first time, an important physiological role for Fer kinase in the innate immune response in the gut. We have demonstrated that in response to systemic LPS challenge, leucocyte recruitment to the lung and intestine is enhanced in the absence of Fer kinase and is associated with exacerbated epithelial barrier dysfunction. LPS induced epithelial barrier dysfunction was completely abolished by depleting circulating neutrophils in wild-type mice but was only partially attenuated by neutrophil depletion in FerDR mice. Our data provide compelling evidence for an important physiological role for Fer in regulating intestinal epithelial barrier integrity in response to LPS challenge, and suggest its involvement in both neutrophil dependent and neutrophil independent mechanisms.

In this study, neutrophil accumulation in the lung following systemic challenge was exacerbated in FerDR mice. This may suggest that Fer kinase activity plays a role in neutrophil rigidity/deformity as it has previously been suggested that LPS induced neutrophil sequestration in the lung is due, in part, to increased neutrophil stiffness mediated by F-actin reorganisation. ${ }^{35}{ }^{36}$ In addition, our data suggest that Fer in wild-type mice may play a role in inhibiting leucocyte adherence on the endothelial surface of intestinal venules. However, significant enhancement in leucocyte migration into intestinal tissue was not observed in FerDR mice although there was a trend in that direction for both neutrophils and lymphocytes. This is in contrast with the massive leucocyte migration observed in response to local LPS challenge in muscle. ${ }^{26}$ Recruitment in this present study was most likely muted due to systemic activation recruiting large numbers of neutrophils to the lungs. In addition, our preliminary data using an in vitro cell migration assay ${ }^{37}$ has demonstrated that neutrophil chemotaxis towards formylmethionylleucylphenylalanine is enhanced in the absence of Fer kinase activity and would support a role for Fer within the neutrophil in cell movement (data not shown). Taken together these data strongly suggest that Fer acts within neutrophils to influence cytoskeletal changes and cell movement.

An additional role for Fer PTK within the endothelial cells cannot be discounted. For example, Fer may be involved in expression of one or more adhesion molecules known to be involved in rolling ( $\mathrm{E}$ and $\mathrm{P}$ selectin) and adhesion (intercellular adhesion molecule 1 and vascular cell adhesion molecule 1), which are increased in the intestinal microvasculature after systemic LPS. ${ }^{38}$ Furthermore, a role in endothelial cells that regulates leucocyte transmigration can be inferred from recent evidence that Fer can phosphorylate PECAM-1, and may therefore be involved in downstream signalling leading to cytoskeletal rearrangements. ${ }^{23}$ PECAM-1 functions as an adhesive and signalling molecule known to play a prominent role in the transmigration of leucocytes across endothelium. ${ }^{39}$ Our previous work demonstrated that endothelial barrier permeability remained intact in FerDR mice after antigen challenge in sensitised mice, ${ }^{26}$ suggesting that the role of Fer within the endothelium is stimulus specific. The role of PECAM-1 phosphorylation by Fer in regulating leucocyte transmigration remains to be determined.

In this study, LPS challenge increased ${ }^{51} \mathrm{Cr}$-EDTA clearance significantly (2.5-fold) by four hours in wild-type mice, and was associated with a mild inflammatory response in the tissue, as illustrated by histological observations. This dose of LPS is significantly lower than the $\mathrm{LD}_{50}{ }^{28}$ and these mice would in all likelihood recover from this challenge. In contrast, the same challenge in FerDR mice induced a 710-fold increase in barrier dysfunction which was notably more severe histologically. Similar fold increases have previously been reported in a model of experimental colitis. ${ }^{31}$ It is important to note that epithelial barrier integrity was intact in FerDR mice under control conditions, and exacerbated barrier dysfunction was noted only after LPS challenge. Neutrophils are key leucocytes recruited during the innate immune response and have previously been shown to cause epithelial barrier dysfunction in other models of intestinal inflammation. ${ }^{24} 40$ Therefore, we investigated whether Fer was acting within neutrophils to induce the exacerbated epithelial barrier dysfunction. Using a monoclonal antibody to deplete circulating neutrophils, we completely abolished LPS induced barrier dysfunction in wild-type mice whereas the same protocol in FerDR mice could only partly inhibit the LPS induced dysfunction. A further reduction in LPS induced dysfunction was not observed on chronic antineutrophil antibody treatment to deplete the resident neutrophil population (data not shown), suggesting that the remaining dysfunction was due to neutrophil independent mechanism(s). Interestingly, neutrophil dependent ${ }^{51} \mathrm{Cr}$-EDTA clearance in FerDR mice was approximately double that observed in wild-type mice. As intestinal neutrophil numbers were not significantly different in FerDR mice, compared with wildtype mice, our data would suggest that neutrophils deficient in Fer kinase activity had an enhanced ability to cause damage to the epithelial barrier, perhaps through enhanced release of proteases or reactive oxygen metabolites. 
It is unclear at this point which cells are involved in neutrophil independent LPS induced epithelial barrier permeability. One possibility is mast cells, which have previously been shown to be involved in intestinal barrier dysfunction during inflammation associated with ischaemia reperfusion, ${ }^{41}{ }^{42}$ stress and hypersensitivity, ${ }^{43}$ and parasitic infections. ${ }^{44}$ In addition, we recently demonstrated a role for Fer in mast cell function through regulation of p38 mitogen activated protein kinase ${ }^{14}$ in response to antigen. However, in that study, mast cell migration and not mediator release was affected by the absence of Fer kinase activity. Another potential site of action might be within the epithelial cell itself. There is a growing body of evidence that suggests a role for Fer in association with adherens junctions and cell-cell contacts, ${ }^{19-23}$ and therefore a role within tight junction complexes in intestinal epithelial cells is an exciting possibility to be explored.

In summary, we have demonstrated in this study that Fer is involved in regulating neutrophil recruitment (adhesion) to the gut and plays a key role in maintaining intestinal epithelial barrier integrity following LPS challenge. Our data further illustrate that Fer can act through both neutrophil dependent and neutrophil independent mechanisms to modulate intestinal barrier permeability in vivo.

\section{ACKNOWLEDGEMENTS}

This work was supported by operating funds from the Canadian Institutes of Health Research (CIHR) and the Crohn's and Colitis Foundation of Canada (CCFC). We would like to thank Dr Rui Zhang for his contribution to this manuscript.

\section{Authors' affiliations}

W Qi, K V J Ebbert, D-M McCafferty, Gastrointestinal Research Group, Department of Physiology and Biophysics, University of Calgary, Calgary, Alberta, Canada

A W B Craig, Department of Biochemistry, Queen's University, Kingston, Ontario, Canada

P A Greer, Department of Pathology and Molecular Medicine and Division of Cancer Biology and Genetics, Queen's University Cancer Research Institute, Queen's University, Kingston, Ontario, Canada Conflict of interest: None declared.

\section{REFERENCES}

1 Warren HS, Chedid LA. Strategies for the treatment of endotoxemia: significance of the acute-phase response. Rev Infect Dis 1987;9:S630-8.

2 Grisham MB, Gaginella TS, von Ritter C, et al. Effects of neutrophil-derived oxidants on intestinal permeability, electrolyte transport, and epithelial cell viability. Inflammation 1990;14:531-42.

3 Arndt H, Kubes P, Granger DN. Involvement of neutrophils in ischemiareperfusion injury in the small intestine. Klinische Wochenschrift 1991;69:1056-60

4 Kubes $\mathbf{P}$, Hunter JA, Granger DN. Ischemia/reperfusion-induced feline intestinal dysfunction: importance of granulocyte recruitment. Gastroenterology 1992; 103:807-12.

5 Kruidenier L, Kuiper I, Lamers CB, et al. Intestinal oxidative damage in inflammatory bowel disease: semi-quantification, localization, and association with mucosal antioxidants. J Pathol 2003;201:28-36.

6 Salvemini D, Riley DP, Lennon PJ, et al. Protective effects of a superoxide dismutase mimetic and peroxynitrite decomposition catalysts in endotoxininduced intestinal damage. Br J Pharmacol 1999;127:685-92.

7 Letwin K, Yee SP, Pawson T. Novel protein-tyrosine kinase cDNAs related to $\mathrm{fps} / \mathrm{fes}$ and eph cloned using anti-phosphotyrosine antibody. Oncogene 1988;3:621-7.

8 Hao QL, Heisterkamp N, Groffen J. Isolation and sequence analysis of a novel human tyrosine kinase gene. Mol Cell Biol 1989:9:1587-93.

9 Robinson DR, Wu YM, Lin SF. The protein tyrosine kinase family of the human genome. Oncogene 2000;19:5548-57.

$10 \mathrm{Kim} \mathrm{L,} \mathrm{Wong} \mathrm{TW.} \mathrm{The} \mathrm{cytoplasmic} \mathrm{tyrosine} \mathrm{kinase} \mathrm{FER} \mathrm{is} \mathrm{associated} \mathrm{with} \mathrm{the}$ catenin-like substrate pp1 20 and is activated by growth factors. Mol Cell Biol 1995; 15:4553-61

11 Craig AW, Zirngibl R, Williams K, et al. Mice devoid of fer protein-tyrosine kinase activity are viable and fertile but display reduced cortactin phosphorylation. Mol Cell Biol 2001;21:603-13.

12 Iwanishi $M$. Overexpression of Fer increases the association of tyrosinephosphorylated IRS-1 with P85 phosphatidylinositol kinase via SH2 domain of Fer in transfected cells. Biochem Biophys Res Commun 2003;311:780-5.
13 Sangrar W, Gao Y, Bates B, et al. Activated Fps/Fes tyrosine kinase regulates erythroid differentiation and survival. Exp Hematol 2004;32:935-45.

14 Craig AW, Greer PA. Fer kinase is required for sustained p38 kinase activation and maximal chemotaxis of activated mast cells. Mol Cell Biol 2002;22:6363-74

15 Senis YA, Sangrar W, Zirngibl RA, et al. Fps/Fes and Fer non-receptor protein-tyrosine kinases regulate collagen- and ADP-induced platelet aggregation. J Thromb Haemost 2003;1:1062-70.

16 Penhallow RC, Class K, Sonoda H, et al. Temporal activation of nontransmembrane protein-tyrosine kinases following mast cell FceRI engagement. J Biol Chem 1995;270:23362-5.

17 Kapus A, Di Ciano C, Sun J, et al. Cell volume-dependent phosphorylation of proteins of the cortical cytoskeleton and cell-cell contact sites. The role of Fyn and FER kinases. J Biol Chem 2000;275:32289-98.

$18 \mathrm{Kim} \mathrm{L}$, Wong TW. Growth factor-dependent phosphorylation of the actinbinding protein cortactin is mediated by the cytoplasmic tyrosine kinase FER. J Biol Chem 1998;273:23542-8.

19 Rosato R, Veltmaat JM, Groffen J, et al. Involvement of the tyrosine kinase fer in cell adhesion. Mol Biol Cell 1998;18:5762-70.

20 Arregui C, Pathre P, Lilien J, et al. The nonreceptor tyrosine kinase fer mediates cross-talk between $\mathrm{N}$-cadherin and betal-integrins. J Cell Biol 2000;149:1263-74.

21 Li H, Leung TC, Hoffman S, et al. Coordinate regulation of cadherin and integrin function by the chondroitin sulfate proteoglycan neurocan. J Cell Biol 2000;149:1275-88.

22 Xu G, Craig AW, Greer P, et al. Continuous association of cadherin with betacatenin requires the non-receptor tyrosine-kinase Fer. J Cell Sci 2004; 117:3207-19.

23 Kogata N, Masuda M, Kamioka Y, et al. Identification of Fer tyrosine kinase localized on microtubules as a platelet endothelial cell adhesion molecule-1 phosphorylating kinase in vascular endothelial cells. Mol Biol Cell 2003; 14:3553-64.

24 Schenkel AR, Chew TW, Muller WA. Platelet endothelial cell adhesion molecule deficiency or blockade significantly reduces leukocyte emigration in a majority of mouse strains. J Immunol 2004;173:6403-8.

25 Maas M, Stapleton M, Bergom C, et al. Endothelial cell PECAM-1 confers protection against endotoxic shock. Am J Physiol Heart Circ Physiol 2005;288:H159-64.

26 McCafferty DM, Craig AW, Senis YA, et al. Absence of Fer protein-tyrosine kinase exacerbates leukocyte recruitment in response to endotoxin. J Immunol 2002;168:4930-5

27 Andonegui G, Goyert SM, Kubes P. Lipopolysaccharide-induced leukocyteendothelial cell interactions: a role for CD14 versus toll-like receptor 4 within microvessels. J Immunol 2002;169:2111-19.

28 Purswani MU, Eckert SJ, Arora HK, et al. Effect of ciprofloxacin on lethal and sublethal challenge with endotoxin and on early cytokine responses in a murine in vivo model. J Antimicrob Chemother 2002;50:51-8.

29 Arndt H, Kubes P, Grisham MB, et al. Granulocyte turnover in the feline intestine. Inflammation 1992;16:549-59.

30 Massberg S, Eisenmenger S, Enders G, et al. Quantitative analysis of small intestinal microcirculation in the mouse. Res Exp Med 1998;198:23-5.

31 McCafferty DM, Miampamba M, Sihota E, et al. Role of inducible nitric oxide synthase in trinitrobenzene sulphonic acid induced colitis in mice. Gut 1999:45:864-73.

32 Krawisz JE, Sharon P, Stenson WF. Quantitative assay for acute inflammation based on myeloperoxidase activity: assessment of inflammation in the rat and hamster models. Gastroenterology 1984;87:1344-50.

33 Kerfoot SM, Kubes P. Overlapping roles of P-selectin and alpha 4 integrin to recruit leukocytes to the central nervous system in experimental autoimmune encephalomyelitis. J Immunol 2002;169:1000-6.

34 Hickey MJ, Issekutz AC, Reinhardt PH, et al. Endogenous interleukin-10 regulates hemodynamic parameters, leukocyte-endothelial cell interactions, and microvascular permeability during endotoxemia. Circ Res 1998;83:1124-31

35 Erzurum SC, Downey GP, Doherty DE, et al. Mechanisms of lipopolysaccharide-induced neutrophil retention. Relative contributions of adhesive and cellular mechanical properties. J Immunol 1992;149:154-62.

36 Tanaka H, Nishino M, Dahms TE. Physiologic responses to small emboli and hemodynamic effects of changes in deformability of polymorphonuclear leukocytes in isolated rabbit lung. Microvasc Res 2002;63:81-90.

37 Heit B, Kubes P. Measuring chemotaxis and chemokinesis: the under-agarose cell migration assay. Sci STKE 2003;170:PL5.

38 Mori N, Horie Y, Gerritsen ME, et al. Anti-inflammatory drugs and endothelial cell adhesion molecule expression in murine vascular beds. Gut 1999;44:186-95.

39 Jackson DE. The unfolding tale of PECAM-1. FEBS Lett 2003;540:7-14.

40 Parks DA, Bulkley GB, Granger DN. Role of oxygen-derived free radicals in digestive tract diseases. Surgery 1983;94:415-22.

41 Kanwar S, Hickey MJ, Kubes P. Postischemic inflammation: a role for mast cells in intestine but not in skeletal muscle. Am J Physiol 1998;275:G212-18.

42 Kanwar S, Kubes P. Mast cells contribute to ischemia/reperfusion-induced granulocyte infiltration and intestinal dysfunction. Am J Physiol 1994;267:G316-21.

43 Yu LC, Perdue MH. Role of mast cells in intestinal mucosal function: studies in models of hypersensitivity and stress. Immunol Rev 2001;179:61-73.

44 McDermott JR, Bartram RE, Knight PA, et al. Mast cells disrupt epithelial barrier function during enteric nematode infection. Proc Natl Acad Sci U S A 2003;100:7761-6. 